\title{
A Method for Evaluation and Comparison of Parallel Robots for Safe Human Interaction, Applied to Robotic TMS
}

\author{
Jan J. de Jong ${ }^{1}$, Arno H.A. Stienen ${ }^{1,2}$, Volkert van der Wijk ${ }^{3}$, Martijn Wessels ${ }^{1}$, and Herman van der Kooij ${ }^{1,4}$.
}

\begin{abstract}
Transcranial magnetic stimulation (TMS) is a noninvasive method to modify behaviour of neurons in the brain. TMS is applied by running large currents through a coil close to the scalp. For consistent results it is required to maintain the coil position within millimetres of the targeted location, but natural head sway and practitioner fatigue may hinder this.

Serial robots are currently used to assist the application of TMS. However, their low stiffness limits the performance and their high moving mass limits the operating speeds for safety reasons. Since 6-DOF parallel robots combine high stiffness with low moving mass, they have potential for fast, safe and accurate positioning required for TMS during activities.

For choosing the safest parallel manipulator, we developed an evaluation method using robotic safety criteria, including motor speed, motor acceleration, force transmission, and workspace accuracy. The method is applied for evaluation and comparison of the Delta robot with rotation head, the Hexaglide robot, and the Hexa robot. The Hexa robot shows to have the best safety characteristics.

This paper also presents the design and evaluation of four controller strategies for the Hexa robot. These strategies include the application of a straightforward PID control (PID), a PID control with Jacobian Feed-Forward (PID J), a PID control with a Spring Force Control (PID $\mathrm{O}^{+}$) and a combination of these (PID J O ${ }^{+}$).
\end{abstract}

\section{INTRODUCTION}

Transcranial magnetic stimulation (TMS) is a non-invasive method which invokes de- or hyper-polarization of the neurons of the brain. TMS is used for diagnosis and treatment of a wide variety of neurological pathologies $[1,2]$. The magnetic stimulation is generated by running large electrical currents through an iron-centre coil held close to scalp. For consistent results over repeated stimulations it is needed to maintain the position of the coil centre within a few millimetres of the targeted location. Natural head sway of the subject undergoing TMS and fatigued arms of the practitioner due to the heavy coil can interfere with the fulfilment of this requirement.

To increase the accuracy and repeatability of TMS and to reduce the strain on the clinician, we wish to develop a robotic system for TMS on subjects in motion, for example

Supporting grants: PIDON (NL): PID082046.

In collaboration with ANT Neuro B.V., Enschede, NL

${ }^{1}$ Laboratory of Biomechanical Engineering, MIRA Institute, University of Twente,Enschede, NL.

2 Department of Physical Therapy and Human Movement Sciences, Northwestern University, Chicago (IL), USA.

${ }^{3}$ Laboratory of Mechanical Automation and Mechatronics, University of Twente, Enschede, NL

${ }^{4}$ Biomechanical Engineering, Delft University of Technology, Delft, NL. Corresponding author: Arno Stienen (arnostienen@gmail.com) during upper-extremity exercises and treadmill walking using exoskeletons.

For such a robot the safety is of utmost importance since design requires a powerful robotic arm to operate in close contact with vital parts of the subject (e.g., the head). To reduce the impact of an electronic, mechanical or software failure, the robot is required to have low kinetic energy and low motor power.

Several conventional robots have been used earlier for TMS, such as the NeuroMate (ISS/IMMI, Sacremento, CA) [3,4], Adept Viper s850 (Adept Technology, Inc. Livermore, CA, USA) [5,6], and Kuka KR3 (Ausburg, Germany) [7]. Most systems use optical tracking of the robot and the subjects head to let the robot position the coil over the desired location on the scalp. Only one robot specially designed for TMS application is found in literature [8].

These robots rely on 6-DOF serial manipulators which have a serial chain of rigid links, connected by revolute joints, with actuators located at each joint. These serial robots combine large operational area with high inertia and low stiffness. To guarantee safety during movement in the vicinity of the human head, the kinetic energy of the robots is kept low by the limitation of the maximum speed. With the aim of TMS stimulation for a human in motion, the combination of safety and accurate tracking is even more challenging.

Parallel manipulators with 6-DOF have potential for such tasks as they combine high positional accuracy with low motion inertia: the motors are mounted on the base and connected to the end-effector via low mass (closed-chain) links.

To evaluate the performance of a manipulator, several methods are available. Most methods focus on acquiring the largest singularity-free workspace for a certain manipulator type. The singularity-free workspace can be found numerically by calculating the position at which the determinant or the condition number of the Jacobian [9]. Singularity loci can also be found analytically [10]-[12]. However, large singularity-free workspace or a dimensionless condition number does not give information about the performance of the robot regarding the safety.

The goal of this paper is to propose a method for the evaluation and comparison of manipulators for safe human interaction for a specific task. The method therefore considers characteristics as the required workspace, the accuracy, motor torques, motor speeds, and motor acceleration.

The paper also presents and evaluates four controller strategies for the Hexa manipulator. The strategies presented 
are: PID control in conjunction with Jacobian based feedforward compensation, contact force control, and a combination of these methods.

First the method is presented, then the selected manipulators for evaluation and comparison are described. The manipulators are evaluated and compared and the results are discussed. Subsequently the controller designs are presented and discussed. s

\section{METHOD}

In order to make a quantitative comparison among various robot types or between geometries, a numerical evaluation of the robotic safety is proposed.

The safety of the robot in each pose can by quantified using five Robotic Safety Indexes (RSI): the maximum motor speed, maximum motor acceleration, maximum motor torque, allowed motor error and the condition number of the Jacobian.

Together these five measures give a indication of the kinematic energy on the end-effector, the motor power and the accuracy within the workspace.

The required motor power can be derived from the maximal motor speed and the maximal motor torque. Motor torque also gives an estimation of the reflected mass from the end-effector, making it a measure for the kinetic energy on the end-effector. The allowed motor error gives a measure for the accuracy of the system. The closeness to singularity can be estimated from the condition number and the maximal motor acceleration to show whether the workspace is large enough.

To calculate the RSIs, first the required workspace and end-effector speeds, accelerations, forces and toques are defined. From these end-effector values the resulting motor values (speed, acceleration, torque and error) can be calculated using the Jacobian (Equation 1)-Equation 6). The RSIs are given by the maximum values of these relations.

The Jacobian $(\mathbb{J})$ and its derivative $\mathrm{f}^{1}(\dot{\overline{\mathbb{J}}})$ are found by partial first and second-order differentiation of the end-effector pose to the motor coordinates.

$$
\mathbb{J}(\mathbf{X})=\left[\begin{array}{ccc}
\frac{\delta \Theta_{1}}{\delta X_{1}} & \cdots & \frac{\delta \Theta_{1}}{\delta X_{k}} \\
\vdots & \ddots & \vdots \\
\frac{\delta \Theta_{n}}{\delta X_{1}} & \cdots & \frac{\delta \Theta_{n}}{\delta X_{k}}
\end{array}\right]
$$

Robotic Safety Indexes are given by the evaluation of the five relations:

1) The relation between the motor speeds $(\dot{\Theta})$ and the generalized end-effector velocity $(\dot{\mathbf{X}})$.

$$
\dot{\Theta}=\rrbracket \dot{\mathbf{X}}
$$

\footnotetext{
${ }^{1}$ The $\dot{\overline{\mathbb{J}}}$ is a $\mathrm{n} \times \mathrm{m} \times \mathrm{n}$ matrix, with $n$ is the number of DOFs and $m$ the number of actuators. The calculation each element of $\ddot{\Theta}_{\mathrm{i}}$ is done by matrix multiplication of each page $(\dot{\overline{\bar{J}}})$ with $\dot{\mathbf{x}}$ and $\dot{\mathbf{x}}^{T}$
}

2) The relation between the motor acceleration $(\ddot{\Theta})$ and the end-effector acceleration $(\ddot{\mathbf{X}})$.

$$
\ddot{\mathbf{\Theta}}=\rrbracket \ddot{\mathbf{X}}+\dot{\mathbf{X}}^{T} \dot{\bar{J}} \dot{\mathbf{X}} .
$$

3) The relation between the generalized motor force $(\tau)$ and end-effector force $(\mathcal{F})$.

$$
\boldsymbol{\tau}=\mathbb{J}^{-T} \mathcal{F}
$$

4) The relation between the allowed motor error $\left(\varepsilon_{m}\right)$ and the end-effector pose error $\left(\varepsilon_{e e}\right)$ gives the error transfer. For this RSI the minimal allowable motor error is taken.

$$
\varepsilon_{m}=\mathbb{J} \varepsilon_{e e}
$$

5) The closeness to singularity of the matrix can be calculated by the condition number $\kappa(\mathbb{J})$ of the Jacobian.

$$
\kappa(\mathbb{J})=\|\mathbb{J}\| \cdot\left\|\mathbb{J}^{-1}\right\|
$$

\section{MANIPULATOR DESCRIPTIONS}

The Robotic Safety Indexes method is applied to the evaluation and comparison of three parallel robot concepts of Figure 1, the Delta robot with rotation head, the Hexaglide, and the Hexa robot.

1) Delta robot with rotation head: The Delta robot with rotational head $\left(3-\underline{R} R P_{a} R-\underline{R R R}\right)^{2}$ consists of a parallel 3DOF translational structure (the Delta robot) combined with a 3-DOF rotational head. The actuators of the rotation head are located on the moving platform of the Delta robot. This 6-DOF Delta robot has recently been developed for pickand-place applications [13] and is used as a surgical tool [14].

2) Hexaglide: The 6-DOF parallel manipulator Hexaglide (6-PUS 3 uses linear motors to actuate six legs. The position and orientation of the glider motor can be arranged to create specific workspaces. The Hexaglide is a simple 6-DOF parallel structure as only two universal joints per kinematic chains are used. The manipulator used is for machine milling applications 4

3) Hexa: The Hexa (6-RUS) manipulator [15] consists of six articulated kinematic chains. The upper arms are actuated by rotational motors, which determine the position and orientation of manipulator platform. Two commercial applications of this robot type are known, both use the 6RUS system for actuation of a flight simulator ${ }^{5}$

The 6-DOF parallel manipulator should continuously and accurately positions the coil to follow the motion of the head. To compensate for different subjects and stimulation sites a passive positioning system places this robotic manipulator at a predetermined height and angle. The required range of motion, speed and acceleration for the TMS robot are

\footnotetext{
${ }^{2}$ The technical name for a robot is given by the subsequent joint types. (R:Rotational Joints, P: Prismatic Joint,S:Spherical Joint,U: Universal Joint, $P_{a}$ Parallelogram structure. Actuated joints are underlined)

${ }^{3}$ In literature other nomenclature for the Hexaglide are found: 6-PSU and 6-PSS, the functional behaviour is similar. This also applies for the Hexa.

${ }^{4}$ http://www.iwf.mavt.ethz.ch/

${ }^{5}$ http://www.servos.com, http://www.fidelityflight.com/
} 


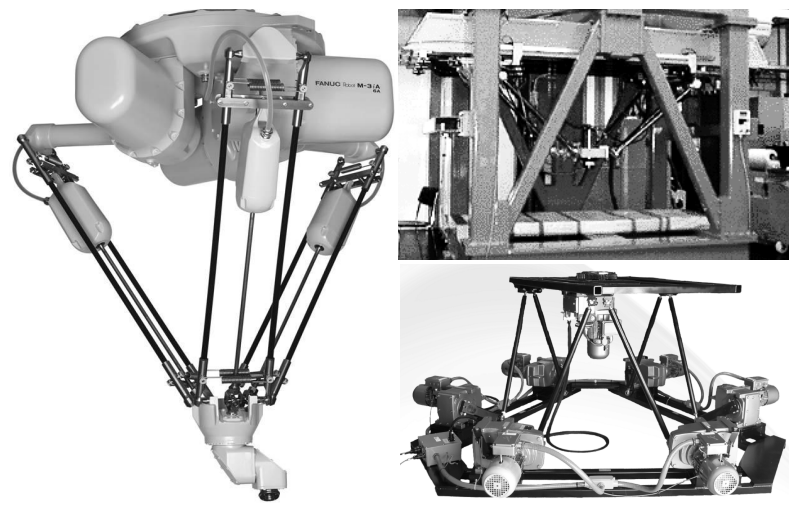

Fig. 1. Three 6-DOF manipulator concepts. Left: Delta robot with rotation head (FANUC M-3iA/6A). Right top: Hexaglide (IWF, ETH, Zurich). Right bottom: Hexa motion platform (Servos \& Simulations Inc. 710-7).

TABLE I

SPECIFICATION OF THE ACTIVE WORKSPACE OF A TMS ROBOT.

\begin{tabular}{cc|cccc}
\hline DOF & Workspace & $\dot{\mathbf{X}}$ & $\ddot{\mathbf{X}}$ & $\mathcal{F}$ & $\boldsymbol{\varepsilon}_{e e}$. \\
\hline $\mathrm{x}$ & 0.10 & 1.50 & 3.00 & 10.5 & 0.5 \\
$\mathrm{y}$ & 0.10 & 1.50 & 3.00 & 10.5 & 0.5 \\
$\mathrm{z}$ & 0.10 & 1.50 & 10.0 & 68.7 & 0.5 \\
$\Phi_{x}$ & 0.51 & 4.73 & 59.7 & 5.37 & 13.0 \\
$\Phi_{y}$ & 0.51 & 4.73 & 59.7 & 5.37 & 13.0 \\
$\Phi_{z}$ & 0.22 & 4.73 & 59.7 & 1.19 & - \\
\hline
\end{tabular}

${ }^{6}$ The workspace is given by the maximal amplitude of the motion in each direction. $\mathrm{X}, \mathrm{y}$, and $\mathrm{z}$ denotes the frontal, lateral and vertical direction. $\Phi_{x}, \Phi_{y}$, and $\Phi_{z}$ are the rotations around the respective axes. The required end-effector velocity is denoted with a $\dot{\mathbf{X}}$, the required acceleration by $\ddot{\mathbf{X}}$, the end-effector wrench is given by $\mathcal{F}$ and end-effector error is given by $\varepsilon_{e e}$. The translational workspace, speed, acceleration, wrench and error are given in, respectively, $m, \mathrm{~m} / \mathrm{s}, \mathrm{m} / \mathrm{s}^{2}, N$ and $\mathrm{mm}$. The rotational values are given in $\mathrm{rad}, \mathrm{rad} / \mathrm{s}, \mathrm{rad} / \mathrm{s}^{2}, \mathrm{Nm}$, and $\mathrm{mrad}$, respectively.

based on literature on head movement [16]-[19] and on experiments with paraplegic subjects wearing the robotic exoskeleton LOPES [20]. The required end-effector wrench is calculated from the maximal acceleration of a typical coil of $3.5 \mathrm{~kg}$. The resulting end-effector values can be found in Table I.

For a fair comparison of the performances, the dimensions of the three robots should be comparable. We chose the dimensions such that distance between base and end-effector is $0.6 \mathrm{~m}$ in outstretched pose. Since the robot arms can be manufactured from lightweight carbon tubes the weight of the moving parts can be neglected with respect to the weight of the coil. For the Delta robot however, an additional weight of $4 \mathrm{~kg}$ for the rotational head has to be taken into account, since the motors of the rotation head are moved during translation. Both the Delta robot and the Hexa use reduction gears with a gear ratio of 1:35.

\section{EVALUATION AND COMPARISON}

For each concept the Jacobian are formulated. The corresponding RSI values are calculated by substituting the values of Table I into Equation 2 Equation 6. The maximal values of each RSI at full rotation inside the workspace are shown in Table II
TABLE II

THE MAXIMUM ROBOTIC SAFETY INDEXES INSIDE THE OPTIMAL WORKSPACE OF THE THREE CONCEPTS AT MAXIMAL ROTATION

\begin{tabular}{llll} 
RS $]^{7}$ & Delta & H-G & Hexa \\
\hline 1. $\ddot{\Theta}_{\max }$ & $1.0710^{3}$ & 4.60 & $2.2210^{3}$ \\
2. $\ddot{\Theta}_{\max }$ & $8.4010^{3}$ & 35.3 & $1.5510^{4}$ \\
3. $\tau_{\max }$ & 1.62 & $9.8610^{5}$ & 1.43 \\
4. $\varepsilon_{\operatorname{man}}$ min & 35.7 & $3.8810^{-5}$ & 7.18 \\
5. $\kappa(\mathbb{J})$ & 16.3 & $1.0710^{5}$ & 127
\end{tabular}

${ }^{7}$ The motor speed $\left(\dot{\Theta}_{\max }\right)$, acceleration $\left(\ddot{\Theta}_{\max }\right)$, torque $\left(\tau_{\max }\right)$ and allowed error $\left(\varepsilon_{m, \min }\right)$ of the Delta Robot (Delta) and Hexa are respectively given in $\mathrm{rad} / \mathrm{s}, \mathrm{rad} / \mathrm{s}^{2}, \mathrm{Nm}$ and $\mathrm{mrad}$ for the Hexaglide (H-G) these are given in $\mathrm{m} / \mathrm{s}, \mathrm{m} / \mathrm{s}^{2}, N \mathrm{~mm}$. The former manipulator types rely on rotational actuators while the latter use linear motors.

Because of limited space, only the motor force plots are shown in Figure 2. The $\mathrm{X}$ - and Y-axis of the plot indicate the position within the slice trough the workspace. The colours in the generalised force plots are normalized on the maximal motor force of the three actuators with similar motor power. The space inside the white box represents the optimal workspace in which the required motor power is minimal.

Table II shows that the Delta robot requires lower motor speed and less acceleration but higher torque to obtain the same end-effector movement than the Hexa. The allowed error motor is larger than that of the other concepts. Figure 2 shows that at the top and near the edges of the range of motion high motor torques are required due to the singularities. The extra mass of the rotation head gives the Delta robot higher kinetic energy, increasing the required torque and reducing the intrinsic safety.

High motor torque values appear through the middle of the workspace of the Hexaglide, revealing a singularity. This singularity occurs during rotation when multiple kinematic chains become co-planar with the end-effector. The singularity decreases the robotics safety strongly, making this robot type unsuitable for the required rotational task.

The XZ-plane of the generalised force plot for the Hexa shows that total range of motion is smaller than that of the Delta Robot and Hexaglide, but large enough for required workspace. The motor torque is more favourable than the other concepts. The force map reveals a singularity outside the active workspace of the Hexa, which does not influence the RSI. Therefore this concept is chosen for optimization and further design, as the kinetic performance is sufficient and the robotic safety is the highest, compared to the Delta robot and the Hexaglide.

Low motor speed, acceleration, torque and error transfer are desirable for a robotic manipulator. However, Equation 2 . Equation 6 show that there is a trade-off among the RSIs. For example, a larger motor arm generally leads to a decrease in the motor speed but also to an increased motor torque and error. 

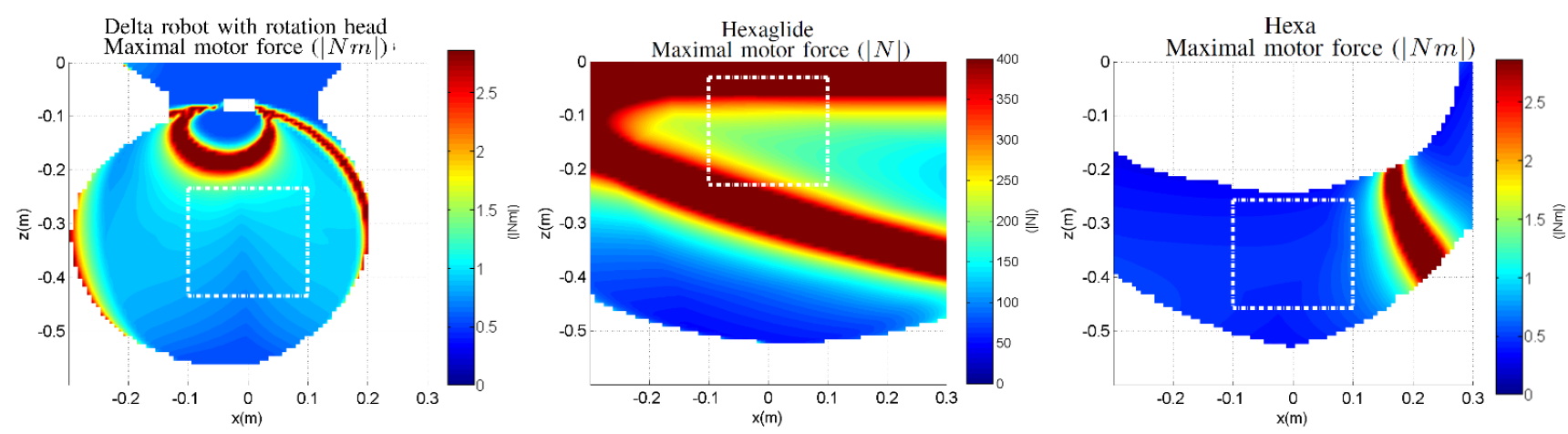

Fig. 2. The XZ plane of the motor generalised force plots show the maximal motor force over the workspace during maximal desired end-effector rotation. The space inside the white box represent the optimal workspace. Middle: Hexaglide. Right: Hexa. For the Delta robot and the Hexa the motor force is given in $N m$. For the Hexaglide the force is given in $N$.

\section{CONTROLLER DESIGN}

Safe tracking of the head can only be achieved by controlling software that is able to detect and handle errors and malfunctions. The controller should be robust enough to reject disturbances and model variations to guarantee stable control over the required workspace.

The input for the controller is derived from head position which is measured with the commercially available Visor TMS neuronavigation system (ANT Neuro B.V., Enschede, The Netherlands).

A spring is placed between the coil and the manipulator to ensure a constant contact between the coil and the head, to prevent collision and to ensure soft human-robot interaction. The contact force between the coil and the head is monitored by measuring the spring deflection.

The dynamic properties of the Hexa mechanism are investigated with the aid of a $3 \mathrm{~d}$ rigid body model in 20sim (Edition 4.1, Controllab Products B.V., Enschede, The Netherlands).

\section{A. Four Controllers}

The disturbances induced by model uncertainties and force perturbations (e.g. variations in skull contact force), are expected to be amplified by the non-linear kinematic and dynamic behaviour of the parallel manipulator. Conventional controller strategies, such as feedback linearisation and compliance control, use joint-spaced dynamic models to calculate required motor torques. For some parallel robots, these jointspaced models are not available and for others they require high computation power [9, p. 286], [21], therefore other strategies are to be used. Four controllers are introduced to compensate for these disturbances and allow adequate response times.

1) PID Control: Under the assumption that the non-linear behaviour is reduced by the reduction gears, a joint spaced PID controller is proposed (see Figure 3). The root locus and Nichols charts of the linearised response of the dynamic model are used to determine the PID values. The controller parameters are aimed at high stability margins. The stability can not be proven rigorously for all the possible poses [22]

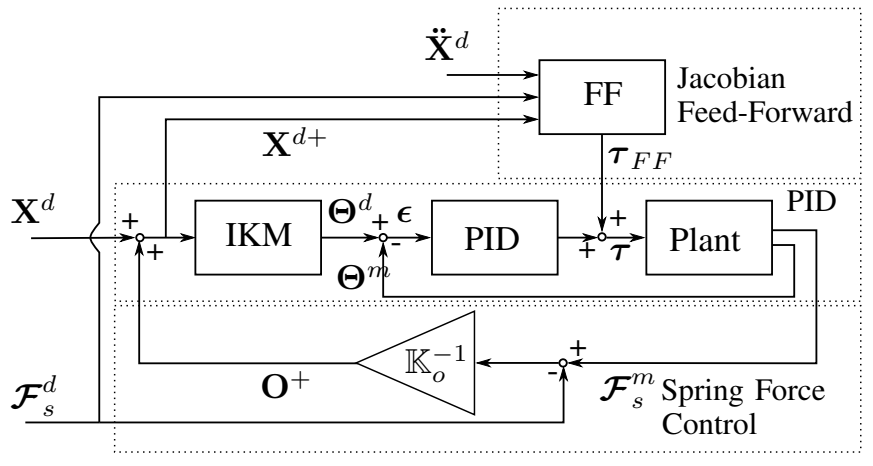

Fig. 3. Different optional elements of the PID controller (PID) with Jacobian Feed-Forward (FF) and Spring Force Control. Here the IKM stands for the Inverse Kinematic Model, Plant stands for the dynamic model of the Hexa including gears and motors. $\mathbf{X}^{d}$ and $\ddot{\mathbf{X}}^{d}$ are the desired pose and acceleration. $\boldsymbol{\Theta}^{d}$ and $\boldsymbol{\Theta}^{m}$ stand for the desired and measured motor angle. $\boldsymbol{\tau}$ and $\boldsymbol{\tau}_{F F}$ are the motor torque and feed-forward torque. $\mathcal{F}_{s}^{d}$ and $\mathcal{F}_{s}^{m}$ denote the desired and measured spring force. $\mathbb{K}_{o}^{-1}$ and $\mathbf{O}^{+}$stand for stiffness matrix and offset, respectively.

and is therefore demonstrated experimentally. The resulting PID controller values are found to be $\mathbb{K}_{P}=8.24, \mathbb{K}_{I}=0.10$, $\mathbb{K}_{D}=1$ and a tameness factor $\chi=0.007$.

2) PID with Jacobian Feed-Forward: The knowledge of the plant (robotic manipulator) can be included into the control for chain decoupling and compensation of known disturbances. However, the dynamic and kinematic models of parallel structures in general require too much computation time to include into an on-line loop, therefore several simplifications and assumptions are introduced here.

It is assumed that Coriolis and centrifugal effects have little or no influence because the required speeds and accelerations are small. The error between the desired and the actual pose is assumed to be small. Further, it is assumed that the total moving mass is located at the position of the coil. These assumptions allow the use of the Jacobian to calculate the required motor torques.

The corresponding control scheme can be found in Figure 3 "Jacobian Feed-Forward". The required end-effector wrench is calculated and substituted into Equation 4 resulting in Equation 7 which produces the feed-forward torque 

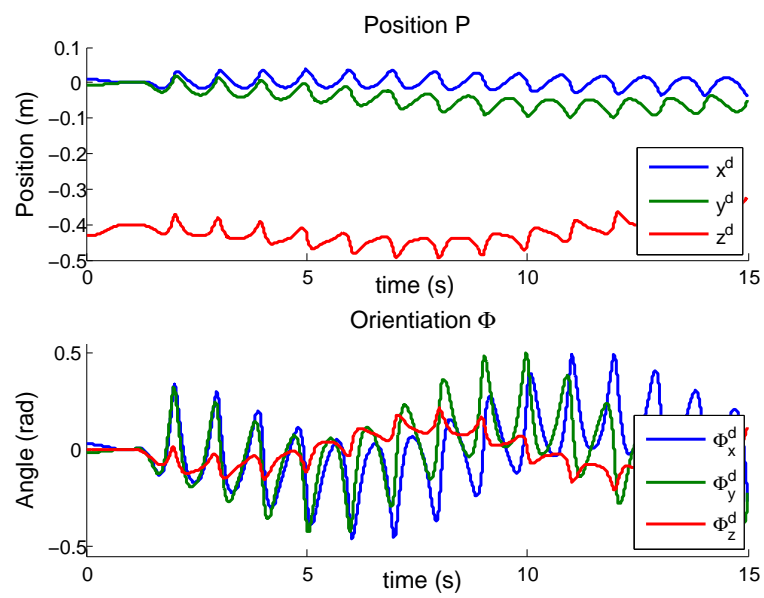

Fig. 4. The trajectory for the evaluation of the controller. The top figure depicts the desired position and the bottom figure shows the desired orientation of the coil.

$\left(\boldsymbol{\tau}_{F F}\right) . \ddot{\mathbf{X}}^{d}$ denotes the desired end-effector acceleration, $\mathbb{M}$ the generalized mass matrix and $\mathbf{G}$ the gravitational acceleration. The desired spring force $\mathcal{F}_{s}^{d}$ is added to this equation to compensate for the spring force disturbance.

$$
J: \boldsymbol{\tau}_{F F}=\mathbb{J}^{-T}\left[\mathbb{M}\left(\ddot{\mathbf{X}}^{d}+\mathbf{G}\right)+\mathcal{F}_{s}^{d}\right]
$$

3) PID with Spring Force Control: It may be expected that the low update frequency and low resolution of the optical tracking system and the flexible connection of the markers to the head will induce some pose error. This error is absorbed by the manipulator spring resulting in an altered spring force.

To obtain a more constant contact force, the measured spring $\mathcal{F}_{c}^{m}$ force is used to change the motion trajectory by an offset $\mathbf{X}^{d+}$, allowing safe stimulation regardless of pose errors (Figure 3. "Spring Force Control"). The control law is given by Equation V-A.3 in which $\mathbb{K}_{o}$ is the pose-dependent virtual stiffness matrix.

$$
O^{+}: \mathbf{X}^{d+}=\mathbf{X}^{d}+\mathbb{K}_{o}^{-1}\left(\mathcal{F}_{s}^{d}-\mathcal{F}_{s}^{m}\right)
$$

4) PID with Jacobian and Spring Force Control: The fourth controller presented here is a combination of the three controllers described earlier. Its control scheme may be found in Figure 3 by combining all optional components.

\section{B. Controller Evaluation}

The four controllers are implemented into 20-sim and connected a $3 \mathrm{~d}$ rigid body model. A typical motion profile for walking is applied to the model. The motion profile consists of a multisine with frequencies between 1 and 10 $\mathrm{Hz}$ and a maximum acceleration of $10 \mathrm{~m} / \mathrm{s}^{2}$ (see Figure 4. The normalized percentage of the tracking error and the spring force are used as measures for the performance of the controllers.

The tracking error and spring force are plotted in Figure 5.
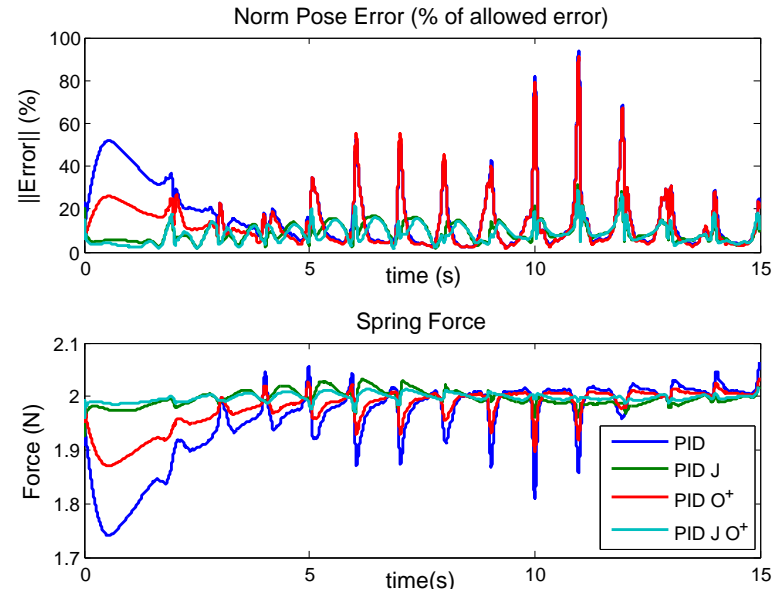

Fig. 5. Top figure shows the normalized error for the four controllers Bottom figure shows resulting spring force. The PID stands for PID controlled, $\mathrm{J}$ and $\mathrm{O}^{+}$denote Jacobian Feed-Forward and Spring Force Control respectively.

It can be observed that the four controllers all follow the trajectory within the error margins of Table I. This suggests that the kinematic model is sufficiently accurate and all the controllers are stable.

Inspection of the error plot of Figure 5 shows that the constant gravitational disturbance is compensated by the integral action of the PID controller. The largest error is observed during maximal acceleration; the dynamic error. This error is pose-dependant and results in changed spring force (Figure 5 bottom).

The addition of the Jacobian Feed-Forward reduces the dynamic and gravitational error strongly, resulting in a more constant spring force.

The Spring Force Control reduces the gravitational error, the spring force variation, but does not result in a reduced dynamic error.

The combination of the Jacobian Feed-Forward and the Spring Force Control results in largest error reduction and the smoothest spring force. Therefore this system is chosen to be most suitable for controlling the Hexa.

\section{DISCUSSION AND CONCLUSION}

The application of TMS during activities such as treadmill walking gives need for robotic guided TMS. For this purpose, a method was presented in this paper to evaluate and compare parallel robots for safe human interaction.

The method considers the motor speed, motor acceleration, motor torque, and workspace accuracy as measures for the robotic safety and quality of the workspace. This method was applied for evaluation and comparison of the Delta robot, Hexaglide and the Hexa. The Hexa manipulator was found to most suitable. be the best of the three for safe human interaction. This is because the kinetic energy and consequently the required motor torque was lower than the other concepts and no singular was found in the required workspace. 
The four controllers for the Hexa manipulator were evaluated on the basis of a dynamic model. The controller using a simple PID control in combination with Jacobian Feed-Forwarding and Spring Force Control gives adequate performance during motion patterns similar to head sway of walking. Simulation showed that the pose accuracy of the robot was sufficient for TMS stimulation.

\section{REFERENCES}

[1] T. Wagner, J. Rushmore, U. Eden, and A. Valero-Cabre, "Biophysical foundations underlying TMS: setting the stage for an effective use of neurostimulation in the cognitive neurosciences." Cortex, vol. 45, no. 9, pp. 1025-34, Oct. 2009.

[2] H. Nollet, "Transcranial magnetic stimulation: review of the technique, basic principles and applications," The Veterinary Journal, vol. 166, no. 1, pp. 28-42, Jul. 2003.

[3] S. Narayana, P. T. Fox, N. Tandon, J. L. Lancaster, J. R. III, M. B. Iyer, and W. Constantine, "Use of neurosurgical robot for aiming and holding in cortical TMS experiments," Neurolmage, vol. 11, no. 5, Supplement 1, pp. S471 - S471, 2000.

[4] J. L. Lancaster, S. Narayana, D. Wenzel, J. Luckemeyer, J. Roby, and P. Fox, "Evaluation of an image-guided, robotically positioned transcranial magnetic stimulation system." Human brain mapping, vol. 22, no. 4, pp. 329-40, Aug. 2004.

[5] S. R. Kantelhardt, T. Fadini, M. Finke, K. Kallenberg, J. Siemerkus, V. Bockermann, L. Matthaeus, W. Paulus, A. Schweikard, V. Rohde, and A. Giese, "Robot-assisted image-guided transcranial magnetic stimulation for somatotopic mapping of the motor cortex: a clinical pilot study." Acta neurochirurgica, vol. 152, no. 2, pp. 333-43, Mar. 2010.

[6] M. Finke, T. Fadini, S. Kantelhardt, A. Giese, L. Matthaus, and A. Schweikard, "Brain-mapping using robotized tms," in Engineering in Medicine and Biology Society, EMBS 2008. 30th Annual International Conference of the IEEE, aug. 2008, pp. 3929 -3932.

[7] L. Matthäus, P. Trillenberg, C. Bodensteiner, A. Giese, and A. Schweikard, "Robotized TMS for motion compensated navigated brain stimulation," in 20th International Computer Assisted Radiology and Surgery Congress, Osaka, Japan, June 2006, pp. 139-141.

[8] C. Lebosse, P. Renaud, B. Bayle, M. de Mathelin, O. Piccin, and J. Foucher, "A robotic system for automated image-guided transcranial magnetic stimulation," in Life Science Systems and Applications Workshop. LISA 2007. IEEE/NIH, nov. 2007, pp. $55-58$.

[9] J.-P. Merlet, Parallel robots, 2nd ed., G. Gladwel, Ed. Springer Dordrecht, The Netherlands, 2006.
[10] E. Macho, O. Altuzarra, E. Amezua, and a. Hernandez, "Obtaining configuration space and singularity maps for parallel manipulators," Mechanism and Machine Theory, vol. 44, no. 11, pp. 2110-2125, Nov. 2009.

[11] I. Bonev and C. Gosselin, "Geometric algorithms for the computation of the constant-orientation workspace and singularity surfaces of a special 6-rus parallel manipulator," in Proceedings of the ASME Design Engineering and Technical Conference, 2002, pp. 505-514.

[12] B. Monsarrat and C. Gosselin, "Workspace analysis and optimal design of a 3-leg 6-DOF parallel platform mechanism," IEEE Transactions on Robotics and Automation, vol. 19, no. 6, pp. 954-966, Dec. 2003.

[13] (2010) Fanuc M-3iA. FANUC Robotics America Corporation. [Online]. Available: http://www.fanucrobotics.com/cmsmedia/datasheets/ M-3iA\%20Series_33.pdf

[14] E. Courteille, D. Deblaise, and P. Maurine, "Design optimization of a Delta-like parallel robot through global stiffness performance evaluation," 2009 IEEE/RSJ International Conference on Intelligent Robots and Systems, pp. 5159-5166, Oct. 2009.

[15] F. Pierrot, P. Dauchez, and A. Fournier, "HEXA: A fast six-DOF fully-parallel robot," in Fifth International Conference on Advanced Robotics, 1991.'Robots in Unstructured Environments', 91 ICAR. IEEE, 1991, pp. 1158-1163.

[16] R. Waters, J. Morris, and J. Perry, "Translational motion of the head and trunk during normal walking," Journal of Biomechanics, vol. 6, no. 2, pp. 167-170, 1973.

[17] A. P. Mulavara, M. C. Verstraete, and J. J. Bloomberg, "Modulation of head movement control in humans during treadmill walking." Gait \& posture, vol. 16, no. 3, pp. 271-82, Dec. 2002.

[18] T. Pozzo, A. Berthoz, L. Lefort, and E. Vitte, "Head stabilization during various locomotor tasks in humans," Experimental brain research, vol. 85, no. 1, pp. 208-217, 1991.

[19] H. B. Menz, S. R. Lord, and R. C. Fitzpatrick, "Acceleration patterns of the head and pelvis when walking on level and irregular surfaces." Gait \& posture, vol. 18, no. 1, pp. 35-46, Aug. 2003.

[20] J. F. Veneman, R. Kruidhof, E. E. G. Hekman, R. Ekkelenkamp, E. H. F. Van Asseldonk, and H. van der Kooij, "Design and evaluation of the LOPES exoskeleton robot for interactive gait rehabilitation." IEEE Transactions on Neural Systems and Rehabilitation Engineering, vol. 15, no. 3, pp. 379-86, Sep. 2007.

[21] R. Boudreau, S. Darenfed, and C. Gosselin, "On the computation of the direct kinematics of parallel manipulators using polynomial networks," Systems, Man and Cybernetics, Part A: Systems and Humans, IEEE Transactions on, vol. 28, no. 2, pp. 213-220, 1998.

[22] Y. Su, B. Duan, and C. Zheng, "Nonlinear PID control of a sixDOF parallel manipulator," in Control Theory and Applications, IEE Proceedings-, vol. 151, no. 1. IET, 2004, pp. 95-102. 